\title{
The Relativistic Rydberg's Formula in Greater Depth and for Any Atom
}

\section{Espen Gaarder Haug}

Norwegian University of Life Sciences, Ås, Norway

Email: espenhaug@mac.com

How to cite this paper: Haug, E.G. (2020) The Relativistic Rydberg's Formula in Greater Depth and for Any Atom. Journal of Modern Physics, 11, 528-534.

https://doi.org/10.4236/jmp.2020.114035

Received: March 8, 2020

Accepted: April 6, 2020

Published: April 9, 2020

Copyright (C) 2020 by author(s) and Scientific Research Publishing Inc. This work is licensed under the Creative Commons Attribution International License (CC BY 4.0).

http://creativecommons.org/licenses/by/4.0/

\begin{abstract}
K. Suto has recently pointed out an interesting relativistic extension of Rydberg's formula. Here we also discuss Rydberg's formula, and offer additional evidence on how one can easily see that it is non-relativistic and therefore a good approximation, at best, when $v \ll c$. We also extend the Suto formula to hold for any atom and examine the formula in detail.
\end{abstract}

\section{Keywords}

Rydberg's Formula, Relativistic Extension, Compton Wavelength

\section{Introduction}

Rydberg's [1] formula is given by

$$
\frac{1}{\lambda}=R_{\infty} Z^{2}\left(\frac{1}{n_{1}^{2}}-\frac{1}{n_{2}^{2}}\right)
$$

where $R_{\infty}$ is the Rydberg's constant, which has a value of 10,973,731.568160 (21) $\mathrm{m}^{-1}$ (NIST CODATA value). Even though the formula is very simple, the intuition behind the formula is hidden in Rydberg's constant and the way the formula is written. To truly understand what Rydberg's formula represents, we will take a close look at what is embedded in the formula.

Rydberg's constant is given by

$$
\begin{gathered}
R_{\infty}=\frac{m_{e} e^{4}}{8 \epsilon_{0}^{2} h^{3} c} \\
R_{\infty}=\frac{\frac{\hbar}{\lambda_{e}} \frac{1}{c}\left(\sqrt{\frac{\hbar}{c}} \sqrt{\alpha} \sqrt{10^{7}}\right)^{4}}{8 \epsilon_{0}^{2} h^{3} c}
\end{gathered}
$$




$$
\begin{gathered}
R_{\infty}=\frac{\frac{\hbar^{3}}{\lambda_{e}} \frac{1}{c^{3}} \alpha^{2}\left(10^{7}\right)^{2}}{8\left(\frac{1}{4 \pi c^{2} 10^{-7}}\right)^{2} h^{3} c} \\
R_{\infty}=\frac{\frac{\hbar^{3}}{\lambda_{e}} \frac{1}{c^{3}} \alpha^{2}}{8 \frac{1}{16 \pi^{2} c^{4}} h^{3} c} \\
R_{\infty}=\frac{1}{2} \frac{\hbar}{h} \frac{1}{\lambda_{e}} \alpha^{2} \\
R_{\infty}=\frac{1}{2} \frac{h}{2 \pi} \frac{1}{h} \alpha^{2} \\
R_{\infty}=\frac{\alpha^{2}}{4 \pi \lambda_{e}}
\end{gathered}
$$

Since the Compton [2] wavelength of the electron is given by ${ }^{1}$

$$
\lambda_{e}=\frac{h}{m_{e} c}
$$

This can be rewritten as

$$
R_{\infty}=\frac{\alpha^{2}}{4 \pi \frac{h}{m_{e} c}}=\frac{\alpha^{2} m_{e} c}{4 \pi h}
$$

This is well known, so we have shown nothing new so far. Let us now replace this in Rydberg's formula, which gives

$$
\begin{gathered}
\frac{1}{\lambda}=\frac{\alpha^{2} m_{e} c}{4 \pi h} Z^{2}\left(\frac{1}{n_{1}^{2}}-\frac{1}{n_{2}^{2}}\right) \\
h \frac{c}{\bar{\lambda}}=Z^{2}\left(\frac{1}{2} m_{e} \frac{\alpha^{2} c^{2}}{n_{1}^{2}}-\frac{1}{2} m_{e} \frac{\alpha^{2} c^{2}}{n_{2}^{2}}\right)
\end{gathered}
$$

where $\frac{\alpha^{2} c^{2}}{n_{1}^{2}}$ can be seen as $v_{1}^{2}$ and $\frac{\alpha^{2} c^{2}}{n_{2}^{2}}$ as $v_{2}^{2}$. In other words, we can write this as

$$
h \frac{c}{\bar{\lambda}}=Z^{2}\left(\frac{1}{2} m_{e} v_{1}^{2}-\frac{1}{2} m_{e} v_{2}^{2}\right)
$$

and since $h \frac{C}{\bar{\lambda}}$ is energy, we can write this as

$$
E=Z^{2}\left(\frac{1}{2} m_{e} v_{1}^{2}-\frac{1}{2} m_{e} v_{2}^{2}\right)
$$

Rydberg's formula is thus the difference in the kinetic energy between two

${ }^{1}$ The original Compton derivation actually gives a non-relativistic Compton wave. That is, it is based on the assumption that the electron is standing still before being hit by photons. For more on the relativistic Compton wave, see [3]. 
electrons (or two states of an electron). However, it is well known that the kinetic energy formula of the form $E_{k}=\frac{1}{2} m v^{2}$ is the first order Taylor series approximation to the relativistic version of the formula. This approximation is only valid when $v \ll c$. In other words, Rydberg's formula is an approximation formula that only holds when the electron moves very slowly as compared to the speed of light. However, it may not be completely obvious or clearly acknowledged that Rydberg's formula is a non-relativistic approximation formula. Standard university textbooks on physics, for example, do not comment that the formula is, in reality, a non-relativistic approximation formula, see [4] and [5], for example.

Turning to a specific case, for a hydrogen atom, it is more precise to use the Rydberg constant

$$
R_{H}=R_{\infty} \frac{m_{P}}{m_{P}+m_{e}}
$$

this means we have

$$
E=Z^{2}\left(\frac{1}{2} m_{e} v_{1}^{2}-\frac{1}{2} m_{e} v_{2}^{2}\right) \frac{m_{P}}{m_{P}+m_{e}}
$$

Before we move on to study relativistic effects, it is also worth mentioning that the Rydberg formula can be rewritten as

$$
\begin{gathered}
h \frac{c}{\bar{\lambda}}=Z^{2}\left(\frac{1}{2} m_{e} \frac{\alpha^{2} c^{2}}{n_{1}^{2}}-\frac{1}{2} m_{e} \frac{\alpha^{2} c^{2}}{n_{2}^{2}}\right) \\
\frac{1}{\bar{\lambda}}=Z^{2}\left(\frac{1}{2} \frac{1}{2 \pi \bar{\lambda}_{e}} \frac{\alpha^{2}}{n_{1}^{2}}-\frac{1}{2} \frac{1}{2 \pi \bar{\lambda}_{e}} \frac{\alpha^{2}}{n_{2}^{2}}\right) \\
\frac{1}{\bar{\lambda}}=Z^{2}\left(\frac{1}{2} \frac{1}{\lambda} \frac{\alpha^{2}}{n_{1}^{2}}-\frac{1}{2} \frac{1}{\lambda_{e}} \frac{\alpha^{2}}{n_{2}^{2}}\right)
\end{gathered}
$$

To set the stage here, all we need to know to obtain the wavelength of the spectra from an atom is the Compton wavelength of the electron, the fine structure constant, and the atomic number. In a recent interesting paper by Suto [6], the author derives a relativistic Rydberg formula that contains the Compton wave of the electron, but he finds it strange that the standard Rydberg formula does not contain the Compton wavelength. In his own words:

"However, Equation (8) for calculating the wavelength of the spectra of a hydrogen atom is strange because it does not include the Compton wavelength of the electron."

where his Equation (8) is the Rydberg formula, here formula 1. But as we can see by rewriting the standard Rydberg formula, the Compton wave of the electron is hidden inside the Rydberg constant, which is a composite constant consisting of more fundamental constants such as the fine structure constant and the Compton wave of the electron. This is clear from Equation (2), where we see the fine 
structure constant and the Compton wave of the electron, as well as $\pi$.

\section{The Relativistic Rydberg Formula}

In the previous section, we observed that Rydberg's formula is a non-relativistic approximation. Recently, Suto [6] has published a relativistic Rydberg formula given by

$$
\frac{1}{\bar{\lambda}}=\frac{1}{\bar{\lambda}_{e}}\left(\left(1-\frac{\alpha^{2}}{n_{1}^{2}}\right)^{-1 / 2}-\left(1-\frac{\alpha^{2}}{n_{2}^{2}}\right)^{-1 / 2}\right)
$$

He also completes a Taylor series expansion series and gets

$$
\frac{1}{\bar{\lambda}}=\frac{1}{\bar{\lambda}_{e}}\left(\left(1-\frac{\alpha^{2}}{2 n_{1}^{2}}-\frac{3 \alpha^{4}}{8 n_{1}^{4}}+\frac{5 \alpha^{6}}{16 n_{1}^{6}}\right)-\left(1-\frac{\alpha^{2}}{2 n_{2}^{2}}-\frac{3 \alpha^{4}}{8 n_{2}^{4}}+\frac{5 \alpha^{6}}{16 n_{2}^{6}}\right)\right)
$$

Here may be a small mistake; we suggest that the correct Taylor expansion should be

$$
\frac{1}{\bar{\lambda}}=\frac{1}{\bar{\lambda}_{e}}\left(\left(1-\frac{\alpha^{2}}{2 n_{1}^{2}}+\frac{3 \alpha^{4}}{8 n_{1}^{4}}+\frac{5 \alpha^{6}}{16 n_{1}^{6}}\right)-\left(1-\frac{\alpha^{2}}{2 n_{2}^{2}}+\frac{3 \alpha^{4}}{8 n_{2}^{4}}+\frac{5 \alpha^{6}}{16 n_{2}^{6}}\right)\right)
$$

In other words, there is a problem with the signs. The error in the Taylor series expansion is likely also the reason the values in the table in his paper are not correct for the prediction of his model. Still, his main result and analysis are correct and we think the relativistic Rydberg formula deserves more attention. For one thing, the Suto formula is only for hydrogen atoms. For a hydrogen atom, the velocity of the electron is very slow, so the difference in predictions between the non-relativistic Rydberg formula and the relativistic formula of Suto is very small and probably not easily evaluated inside the error bounds in measurements.

However, for much heavier elements many of the electrons are moving considerably faster. Here we extend that formula to hold for any element and we get

$$
\begin{gathered}
\frac{h}{\lambda} c=\frac{m_{e} c^{2}}{\sqrt{1-\left(z^{2} \alpha^{2} / n_{1}^{2}\right)}}-m_{e} c^{2}-\frac{m_{e} c^{2}}{\sqrt{1-\left(z^{2} \alpha^{2} / n_{2}^{2}\right)}}+m_{e} c^{2} \\
\frac{h}{\lambda} c=\frac{m_{e} c^{2}}{\sqrt{1-\left(z^{2} \alpha^{2} / n_{1}^{2}\right)}}-\frac{m_{e} c^{2}}{\sqrt{1-\left(z^{2} \alpha^{2} / n_{2}^{2}\right)}} \\
\frac{1}{\bar{\lambda}}=\frac{1}{\bar{\lambda}_{e}}\left(\frac{1}{\sqrt{1-\left(z^{2} \alpha^{2} / n_{1}^{2}\right)}}-\frac{1}{\sqrt{1-\left(z^{2} \alpha^{2} / n_{2}^{2}\right)}}\right)
\end{gathered}
$$

where $z$ is the atom/element number. Table 1 shows predictions from both the non-relativistic Rydberg formula and our relativistic formula for element 1 (Hydrogen) and up to element 137 (Feynmanium). Another interesting aspect here is that the Rydberg formula is somewhat linked to the Bohr model, which is obviously only an approximation. In practice, many predictions are done from quantum mechanics, such as results from the Dirac [7] equation. It is therefore 
Table 1. The table shows the Rydberg formula predictions and the relativistic predictions for the first 137 elements. As we can see, the difference increases between the two models the higher the element number is. Here we are just looking at the case $n_{1}=1$ and $n_{2}=2$.

\begin{tabular}{|c|c|c|c|c|c|c|c|c|c|}
\hline $\begin{array}{c}\text { Atomic } \\
\quad \#\end{array}$ & $\begin{array}{l}\text { Rydberg } \\
\text { formula }\end{array}$ & $\begin{array}{l}\text { Relativistic } \\
\text { formula }\end{array}$ & Diff. & $\begin{array}{c}\text { Diff. } \\
\%\end{array}$ & $\begin{array}{c}\text { Atomic } \\
\#\end{array}$ & $\begin{array}{l}\text { Rydberg } \\
\text { formula }\end{array}$ & $\begin{array}{l}\text { Relativistic } \\
\text { formula }\end{array}$ & Diff. & $\begin{array}{c}\text { Diff. } \\
\%\end{array}$ \\
\hline 1 & 121.5023 & 121.4962 & -0.0061 & $-0.0050 \%$ & 71 & 0.0241 & 0.0144 & -0.0097 & $-67.6 \%$ \\
\hline 2 & 30.3756 & 26.0315 & -4.3440 & $-16.7 \%$ & 72 & 0.0234 & 0.0139 & -0.0096 & $-68.9 \%$ \\
\hline 3 & 13.5003 & 11.0414 & -2.4589 & $-22.3 \%$ & 73 & 0.0228 & 0.0134 & -0.0094 & $-70.2 \%$ \\
\hline 4 & 7.5939 & 6.0710 & -1.5229 & $-25.1 \%$ & 74 & 0.0222 & 0.0129 & -0.0093 & $-71.6 \%$ \\
\hline 5 & 4.8601 & 3.8329 & -1.0272 & $-26.8 \%$ & 75 & 0.0216 & 0.0125 & -0.0091 & $-73.0 \%$ \\
\hline 6 & 3.3751 & 2.6374 & -0.7377 & $-28.0 \%$ & 76 & 0.0210 & 0.0121 & -0.0090 & $-74.5 \%$ \\
\hline 7 & 2.4796 & 1.9247 & -0.5549 & $-28.8 \%$ & 77 & 0.0205 & 0.0116 & -0.0088 & $-76.0 \%$ \\
\hline 8 & 1.8985 & 1.4659 & -0.4326 & $-29.5 \%$ & 78 & 0.0200 & 0.0112 & -0.0087 & $-77.6 \%$ \\
\hline 9 & 1.5000 & 1.1533 & -0.3467 & $-30.1 \%$ & 79 & 0.0195 & 0.0109 & -0.0086 & $-79.2 \%$ \\
\hline 10 & 1.2150 & 0.9308 & -0.2842 & $-30.5 \%$ & 80 & 0.0190 & 0.0105 & -0.0085 & $-80.9 \%$ \\
\hline 11 & 1.0042 & 0.7668 & -0.2373 & $-31.0 \%$ & 81 & 0.0185 & 0.0101 & -0.0084 & $-82.6 \%$ \\
\hline 12 & 0.8438 & 0.6425 & -0.2013 & $-31.3 \%$ & 82 & 0.0181 & 0.0098 & -0.0083 & $-84.4 \%$ \\
\hline 13 & 0.7189 & 0.5460 & -0.1729 & $-31.7 \%$ & 83 & 0.0176 & 0.0095 & -0.0082 & $-86.2 \%$ \\
\hline 14 & 0.6199 & 0.4696 & -0.1503 & $-32.0 \%$ & 84 & 0.0172 & 0.0092 & -0.0081 & $-88.1 \%$ \\
\hline 15 & 0.5400 & 0.4081 & -0.1319 & $-32.3 \%$ & 85 & 0.0168 & 0.0088 & -0.0080 & $-90.1 \%$ \\
\hline 16 & 0.4746 & 0.3579 & -0.1168 & $-32.6 \%$ & 86 & 0.0164 & 0.0085 & -0.0079 & $-92.2 \%$ \\
\hline 17 & 0.4204 & 0.3163 & -0.1042 & $-32.9 \%$ & 87 & 0.0161 & 0.0083 & -0.0078 & $-94.3 \%$ \\
\hline 18 & 0.3750 & 0.2815 & -0.0935 & $-33.2 \%$ & 88 & 0.0157 & 0.0080 & -0.0077 & $-96.5 \%$ \\
\hline 19 & 0.3366 & 0.2521 & -0.0845 & $-33.5 \%$ & 89 & 0.0153 & 0.0077 & -0.0076 & $-98.8 \%$ \\
\hline 20 & 0.3038 & 0.2270 & -0.0768 & $-33.8 \%$ & 90 & 0.0150 & 0.0075 & -0.0075 & $-101.2 \%$ \\
\hline 21 & 0.2755 & 0.2054 & -0.0701 & $-34.1 \%$ & 91 & 0.0147 & 0.0072 & -0.0075 & $-103.7 \%$ \\
\hline 22 & 0.2510 & 0.1867 & -0.0643 & $-34.5 \%$ & 92 & 0.0144 & 0.0070 & -0.0074 & $-106.3 \%$ \\
\hline 23 & 0.2297 & 0.1704 & -0.0593 & $-34.8 \%$ & 93 & 0.0140 & 0.0067 & -0.0073 & $-109.0 \%$ \\
\hline 24 & 0.2109 & 0.1561 & -0.0548 & $-35.1 \%$ & 94 & 0.0138 & 0.0065 & -0.0073 & $-111.8 \%$ \\
\hline 25 & 0.1944 & 0.1436 & -0.0509 & $-35.4 \%$ & 95 & 0.0135 & 0.0063 & -0.0072 & $-114.7 \%$ \\
\hline 26 & 0.1797 & 0.1324 & -0.0473 & $-35.8 \%$ & 96 & 0.0132 & 0.0061 & -0.0071 & $-117.7 \%$ \\
\hline 27 & 0.1667 & 0.1225 & -0.0442 & $-36.1 \%$ & 97 & 0.0129 & 0.0058 & -0.0071 & $-120.9 \%$ \\
\hline 28 & 0.1550 & 0.1136 & -0.0414 & $-36.5 \%$ & 98 & 0.0127 & 0.0056 & -0.0070 & $-124.2 \%$ \\
\hline 29 & 0.1445 & 0.1056 & -0.0389 & $-36.8 \%$ & 99 & 0.0124 & 0.0054 & -0.0070 & $-127.7 \%$ \\
\hline 30 & 0.1350 & 0.0984 & -0.0366 & $-37.2 \%$ & 100 & 0.0122 & 0.0053 & -0.0069 & $-131.3 \%$ \\
\hline 31 & 0.1264 & 0.0919 & -0.0346 & $-37.6 \%$ & 101 & 0.0119 & 0.0051 & -0.0068 & $-135.1 \%$ \\
\hline 32 & 0.1187 & 0.0860 & -0.0327 & $-38.0 \%$ & 102 & 0.0117 & 0.0049 & -0.0068 & $-139.1 \%$ \\
\hline 33 & 0.1116 & 0.0806 & -0.0310 & $-38.4 \%$ & 103 & 0.0115 & 0.0047 & -0.0067 & $-143.3 \%$ \\
\hline 34 & 0.1051 & 0.0757 & -0.0294 & $-38.8 \%$ & 104 & 0.0112 & 0.0045 & -0.0067 & $-147.7 \%$ \\
\hline
\end{tabular}


Continued

\begin{tabular}{|c|c|c|c|c|c|c|c|c|c|}
\hline 35 & 0.0992 & 0.0712 & -0.0280 & $-39.3 \%$ & 105 & 0.0110 & 0.0044 & -0.0067 & $-152.3 \%$ \\
\hline 36 & 0.0938 & 0.0671 & -0.0267 & $-39.7 \%$ & 106 & 0.0108 & 0.0042 & -0.0066 & $-157.2 \%$ \\
\hline 37 & 0.0888 & 0.0633 & -0.0254 & $-40.2 \%$ & 107 & 0.0106 & 0.0040 & -0.0066 & $-162.4 \%$ \\
\hline 38 & 0.0841 & 0.0598 & -0.0243 & $-40.7 \%$ & 108 & 0.0104 & 0.0039 & -0.0065 & $-167.9 \%$ \\
\hline 39 & 0.0799 & 0.0566 & -0.0233 & $-41.2 \%$ & 109 & 0.0102 & 0.0037 & -0.0065 & $-173.7 \%$ \\
\hline 40 & 0.0759 & 0.0536 & -0.0223 & $-41.7 \%$ & 110 & 0.0100 & 0.0036 & -0.0065 & $-179.8 \%$ \\
\hline 41 & 0.0723 & 0.0508 & -0.0214 & $-42.2 \%$ & 111 & 0.0099 & 0.0034 & -0.0064 & $-186.3 \%$ \\
\hline 42 & 0.0689 & 0.0483 & -0.0206 & $-42.7 \%$ & 112 & 0.0097 & 0.0033 & -0.0064 & $-193.3 \%$ \\
\hline 43 & 0.0657 & 0.0459 & -0.0199 & $-43.3 \%$ & 113 & 0.0095 & 0.0032 & -0.0064 & $-200.8 \%$ \\
\hline 44 & 0.0628 & 0.0436 & -0.0191 & $-43.9 \%$ & 114 & 0.0093 & 0.0030 & -0.0063 & $-208.8 \%$ \\
\hline 45 & 0.0600 & 0.0415 & -0.0185 & $-44.4 \%$ & 115 & 0.0092 & 0.0029 & -0.0063 & $-217.3 \%$ \\
\hline 46 & 0.0574 & 0.0396 & -0.0178 & $-45.1 \%$ & 116 & 0.0090 & 0.0028 & -0.0063 & $-226.6 \%$ \\
\hline 47 & 0.0550 & 0.0378 & -0.0172 & $-45.7 \%$ & 117 & 0.0089 & 0.0026 & -0.0062 & $-236.6 \%$ \\
\hline 48 & 0.0527 & 0.0360 & -0.0167 & $-46.3 \%$ & 118 & 0.0087 & 0.0025 & -0.0062 & $-247.4 \%$ \\
\hline 49 & 0.0506 & 0.0344 & -0.0162 & $-47.0 \%$ & 119 & 0.0086 & 0.0024 & -0.0062 & $-259.2 \%$ \\
\hline 50 & 0.0486 & 0.0329 & -0.0157 & $-47.7 \%$ & 120 & 0.0084 & 0.0023 & -0.0062 & $-272.1 \%$ \\
\hline 51 & 0.0467 & 0.0315 & -0.0152 & $-48.4 \%$ & 121 & 0.0083 & 0.0021 & -0.0062 & $-286.3 \%$ \\
\hline 52 & 0.0449 & 0.0301 & -0.0148 & $-49.1 \%$ & 122 & 0.0082 & 0.0020 & -0.0061 & $-302.0 \%$ \\
\hline 53 & 0.0433 & 0.0289 & -0.0144 & $-49.8 \%$ & 123 & 0.0080 & 0.0019 & -0.0061 & $-319.5 \%$ \\
\hline 54 & 0.0417 & 0.0277 & -0.0140 & $-50.6 \%$ & 124 & 0.0079 & 0.0018 & -0.0061 & $-339.1 \%$ \\
\hline 55 & 0.0402 & 0.0265 & -0.0136 & $-51.4 \%$ & 125 & 0.0078 & 0.0017 & -0.0061 & $-361.3 \%$ \\
\hline 56 & 0.0387 & 0.0255 & -0.0133 & $-52.2 \%$ & 126 & 0.0077 & 0.0016 & -0.0061 & $-386.6 \%$ \\
\hline 57 & 0.0374 & 0.0244 & -0.0130 & $-53.0 \%$ & 127 & 0.0075 & 0.0015 & -0.0061 & $-415.8 \%$ \\
\hline 58 & 0.0361 & 0.0235 & -0.0126 & $-53.8 \%$ & 128 & 0.0074 & 0.0013 & -0.0061 & $-450.0 \%$ \\
\hline 59 & 0.0349 & 0.0226 & -0.0123 & $-54.7 \%$ & 129 & 0.0073 & 0.0012 & -0.0061 & $-490.7 \%$ \\
\hline 60 & 0.0338 & 0.0217 & -0.0121 & $-55.6 \%$ & 130 & 0.0072 & 0.0011 & -0.0061 & $-540.2 \%$ \\
\hline 61 & 0.0327 & 0.0209 & -0.0118 & $-56.6 \%$ & 131 & 0.0071 & 0.0010 & -0.0061 & $-602.1 \%$ \\
\hline 62 & 0.0316 & 0.0201 & -0.0115 & $-57.5 \%$ & 132 & 0.0070 & 0.0009 & -0.0061 & $-682.3 \%$ \\
\hline 63 & 0.0306 & 0.0193 & -0.0113 & $-58.5 \%$ & 133 & 0.0069 & 0.0008 & -0.0061 & $-791.8 \%$ \\
\hline 64 & 0.0297 & 0.0186 & -0.0111 & $-59.5 \%$ & 134 & 0.0068 & 0.0006 & -0.0061 & $-953.2 \%$ \\
\hline 65 & 0.0288 & 0.0179 & -0.0108 & $-60.6 \%$ & 135 & 0.0067 & 0.0005 & -0.0062 & $-1224.9 \%$ \\
\hline 66 & 0.0279 & 0.0173 & -0.0106 & $-61.7 \%$ & 136 & 0.0066 & 0.0003 & -0.0062 & $-1835.0 \%$ \\
\hline 67 & 0.0271 & 0.0166 & -0.0104 & $-62.8 \%$ & 137 & 0.0065 & 0.0001 & -0.0064 & $-11273.7 \%$ \\
\hline 68 & 0.0263 & 0.0160 & -0.0102 & $-63.9 \%$ & & & & & \\
\hline 69 & 0.0255 & 0.0155 & -0.0101 & $-65.1 \%$ & & & & & \\
\hline 70 & 0.0248 & 0.0149 & -0.0099 & $-66.3 \%$ & & & & & \\
\hline
\end{tabular}


not clear if the relativistic Rydberg formula has much to offer or not, but it is important for anyone interested in physics to know that it is, at best, a good approximation when the velocity of the electron is $v \ll c$.

\section{Conclusion}

Suto has recently published an interesting relativistic version of the Rydberg formula. Here we have added additional evidence and insight on how, after some reformulation, one can easily see that the Rydberg formula is simply a non-relativistic approximation. We have also extended the Suto relativistic formula to hold for any element. For those interested in this area of physics, further exploration may yield additional insights.

\section{Conflicts of Interest}

The author declares no conflicts of interest regarding the publication of this paper.

\section{References}

[1] Rydberg, J.R. (1890) Philosophical Magazine, 29, 331-337. https://doi.org/10.1080/14786449008619945

[2] Compton, A.H. (1923) Physical Review, 21, 210. https://doi.org/10.1103/PhysRev.21.483

[3] Haug, E.G. (2020) Derivation of a Relativistic Compton Wave. https://vixra.org/abs/2002.0523

[4] Walker, J.S. (2010) Physics. 4th Edition, Addison-Wesley, Boston.

[5] Freedman, R.A. and Young, H.D. (2016) University Physics with Modern Physics. 14th Edition, Pearson, London.

[6] Suto, K. (2020) Journal of Modern Physics, 11, 294-303. https://doi.org/10.4236/jmp.2020.112018

[7] Dirac, P.A.M. (1928) The Quantum Theory of the Electron. Proceedings of the Royal Society London A, Vol. 117, 610-624. https://doi.org/10.1098/rspa.1928.0023 